\title{
Anisotropic large deformation of geometrically architectured unfilled silicone membranes
}

\author{
L. Meunier ${ }^{\mathrm{a}, \mathrm{c}, 1}$, G. Chagnon ${ }^{\text {b, * }}$, D. Favier ${ }^{\mathrm{b}}$, L. Orgéas ${ }^{\mathrm{a}, \mathrm{c}}$ \\ ${ }^{a}$ CNRS, 3SR Lab, F-38000 Grenoble, France \\ ${ }^{\mathrm{b}}$ Université de Grenoble/CNRS, TIMC-IMAG UMR 5525, Grenoble, France \\ ${ }^{\mathrm{c}}$ Univ. Grenoble Alpes, 3SR Lab, F-38000 Grenoble, France
}

\begin{abstract}
Many applications, especially in the medical field, need the use of highly deformable membranes with required anisotropic properties. The present work is a contribution towards the processing, characterisation and modelling of anisotropic hyperelastic membranes. An unfilled silicone rubber with perfect hyperelastic behaviour is used. The anisotropy is generated by adding orientated crenels on the upper and lower surfaces of thin membranes during their elaboration. The influence of the relative orientation of the crenels on the mechanical response is characterised by performing tensile tests combined with kinematic field measurements by Digital Image Correlation. Two modellings are proposed. First, a simple analytical equivalent membrane model is proposed aiming to represent the behaviour of the architectured silicone membranes without any more parameter than those used in the hyperelastic constitutive equation of the silicone rubber. Second, the effective properties of the membranes are obtained by an a homogenisation approach with multiple scale asymptotic expansions written in the framework of hyperelasticity and by solving localisation problems on Representative Elementary Volumes with a finite element software. Finally, the experimental results are compared with predictions of two modelling, both approaches are equally efficient to describe them.
\end{abstract}

\section{Introduction}

Architectured materials take a place more and more important in many applications (Dunlop and Brechet, 2009) due to their specific properties including mechanical properties (Bouaziz et al., 2008; Bouaziz, 2013; Fleck et al., 2010), especially in medical applications. They can be used at different scales (Bréchet and Embury, 2013), from the molecular scale (Pouget et al., 2010) with biomolecules (Patterson et al., 2010) to materials for tissues (Kidoaki et al., 2008; Ma, 2008; Chen et al., 2008). The ability of materials to exhibit anisotropic behaviour is a key point to mimic living tissues. These materials are inspired from industrial materials, since there exist many different microstructures classically studied as foams or cellular materials (Gibson and Ashby, 1982, 1988; Jacobsen et al., 2008; Melchels et al., 2010) or composite materials (Laszczyk

\footnotetext{
* Corresponding author. Fax: +33476768844.

E-mail address: gregory.chagnon@imag.fr (G. Chagnon).

1 In memory of Luc Meunier.
}

et al., 2008) like manufactured woven or non-woven two dimensional (2D) textiles. The design of such structures permits to obtain very particular and non classical properties (Ashby, 2013).

These materials are often anisotropic due to the orientation of the material microstructure in some privileged directions. The knowledge of these properties is a key point for their use in real structures. Such structures are usually periodic with a Representative Elementary Volume (REV) that is repeated in space. The design of such structures needs the use of finite element (FE) calculations to optimise them. But the creation of the geometry and the mesh of such structures necessitates a large number of elements which leads to non-usable FE models, as the calculation time is not compatible with an optimising process.

Instead of considering the geometrical details of the architectured material, it is preferred to use an equivalent constitutive equation that permits to describe the mechanical behaviour of the REV of the architectured material. The best way is to propose a simple analytical form that can easily be implemented in finite element codes. This allows to optimise a structure and to take into account the microstructures of the architectured material without a 
disproportionate number of elements. In this way, many works were developed to propose an equivalent constitutive equation for architectured materials. The reader can for example refer to Talbi et al. (2009) for cardboard, Hard af Segerstad et al. (2008) for honeycombs, Toll (1998) for entangled fibrous materials or Holzapfel et al. (2000) for living tissues.

The difficulty is often to validate analytical model on experimental results because of non-linear phenomena often present in these materials as for example plasticity, damage or viscoelasticity. In this paper, it is proposed to develop an architectured anisotropic hyperelastic material based on an unfilled silicone rubber and to write an equivalent constitutive equation easily usable in finite element codes. The paper focuses on a methodology which starts from experimental measures to analytical equations. It details all the steps of an equivalent modelling and permits to validate experimentally an analytical constitutive equation for architectured materials.

In Section 2, the process to elaborate architectured periodic silicone structures is detailed and the experimental devices to fabricate the specimens are presented. In Section 3, experimental results on the silicone rubber constituent material and on the architectured material are presented. In Section 4, an equivalent analytical anisotropic hyperelastic constitutive equation is written by the superposition of the contribution of each element of the REV i.e. the core membrane and the crenels. In Section 5, the theoretical basis for the homogenisation method with multiple scale asymptotic expansions for periodic structures are detailed and implemented in a finite element code to evaluate the equivalent properties of REV of crenellated silicone membrane. In Section 6, the ability of the two modellings to represent experimental data are discussed. Finally, some concluding remarks close the paper.

\section{Experimental procedure}

\subsection{Architectured membranes: design and processing}

To avoid interface problems such as those often encountered in usual fibre reinforced composite materials, an approach based on a unique material and geometrical reinforcement was here preferred.

An unfilled silicone rubber formulation (RTV141, Bluestar Silicones, France) was chosen. The mechanical behaviour of the material was studied by Meunier et al. (2008) and Rey et al. (2013). It was chosen due to its very interesting properties: it allowed the design and the processing of nearly hyperelastic architectured membranes, with reduced hysteresis and Mullins effect on the contrary of filled silicone rubber (Machado et al., 2010, 2012a, b). Compared with the silicone studied in Meunier et al. (2008), the curing time was here increased from 150 to $360 \mathrm{~min}$ in order to strengthen the membranes and to saturate the reticulation (Rey et al., 2013). After processing, a very weak Mullins effect was observed but it could be considered as negligible.

The upper and the lower external surfaces of the flat silicone membranes were ascribed geometrical motifs, i.e. two lattices of parallel crenels (see Fig. 1 for the dimensions of the crenels) with a relative orientation $2 \alpha$ between the upper and lower crenels (see Fig. 2). This type of architecture was chosen because it ensures an anisotropic response of the membranes and no out of plane bending modes while in-plane stretching can be observed.

Such architectured membranes were processed by injection moulding. For that purpose, a specific mould was designed, as shown in Fig. 3. It is composed of two $16 \mathrm{~cm}$ diameter circular crenellated metallic plates which are locked on each side of a holed plate. The holed plate is equipped with injection gates in order to inject the uncured silicone into the mould before its curing. The two crenellated plates can be fixed separately in any wished angular

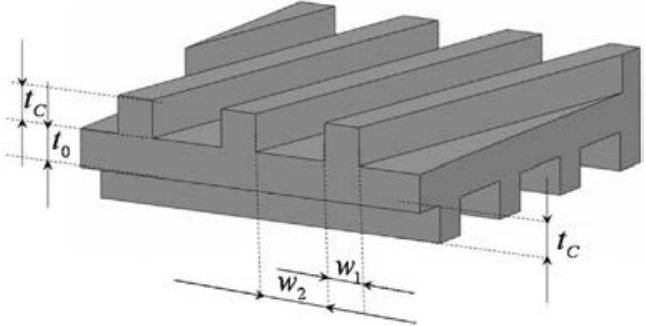

Fig. 1. Sketch and dimensions of the studied architectured membranes: $t_{0}=t_{c}=1 \mathrm{~mm}$, $w_{2}=2 w_{1}=2 \mathrm{~mm}$.

position. Thus, a single apparatus permits to elaborate many architectured mesostructures, by adjusting the angle $2 \alpha$ between the upper and lower crenels directions. Finally, the mould was used to process circular crenellated plates, with six angles $2 \alpha: 25^{\circ}, 45^{\circ}$, $65^{\circ}, 115^{\circ}, 135^{\circ}$ and $155^{\circ}$. An example is shown in Fig. 3.

\subsection{Mechanical characterisation}

Tensile, pure shear and bulge tests were performed on the bulk silicone rubber to analyse and model its behaviour, as in Meunier et al. (2008). The architectured membranes were also subjected to tensile loadings. For that purpose, rectangular specimens were cut from the processed crenellated plates. The specimen cutting was such that their initial length $L_{0}$ was parallel to the bisection of the crenels, as shown in Fig. 2. Likewise, by taking into account the constraints imposed by the processing route and by the testing conditions, the maximum number of crenels along the width of the specimen was used. For angles of $25^{\circ}, 45^{\circ}, 65^{\circ}, 115^{\circ}$, seven crenels were used, but larger values of the angles lead to very large sample which were not compatible with tensile test hypothesis, thus only five crenels were used for $135^{\circ}$ and three for $155^{\circ}$.

Whatever the tested sample, the tensile and pure shear tests were realised on a universal mechanical testing machine (MTS 4M). This machine was equipped with a $250 \mathrm{~N}$ load cell used to measure the tensile force $F$. Together with the initial sample width $h_{0}, F$ was used to estimate the nominal tension $T=F / h_{0}$ required to deform the membranes.

Furthermore, prior all tests, the external surfaces of each specimen were coated with a random pattern made of small speckles (it can be seen on the surface of the specimen in Fig. 2) in order to allow the measurement of local strain fields on the samples surface with digital image correlation (DIC). For that purpose, the 7D DIC software was also used for the tensile and pure shear tests (Vacher et al., 1999). For the bulge tests (Machado et al., 2012a, b), stereo digital image correlation (SDIC, Orteu (2009)) was used, only the top of the bulge test was treated, as it represents an equibiaxial loading.

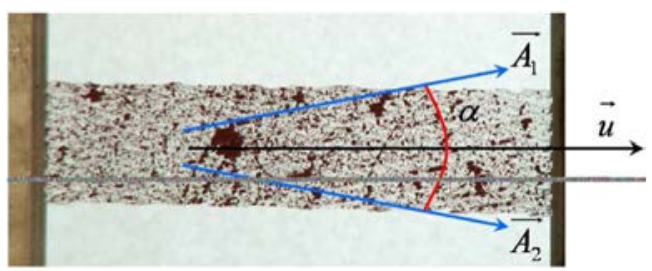

Fig. 2. Tensile rectangular specimen cut from the processed circular plate shown in Fig. 3. Definition of the relative orientation $2 \alpha$ between the upper and lower crenels The specimen is mounted in the gripping system of the tensile testing machine. It is also coated with a random pattern of small speckles to allow DIC measurements (Meunier et al., 2008). 

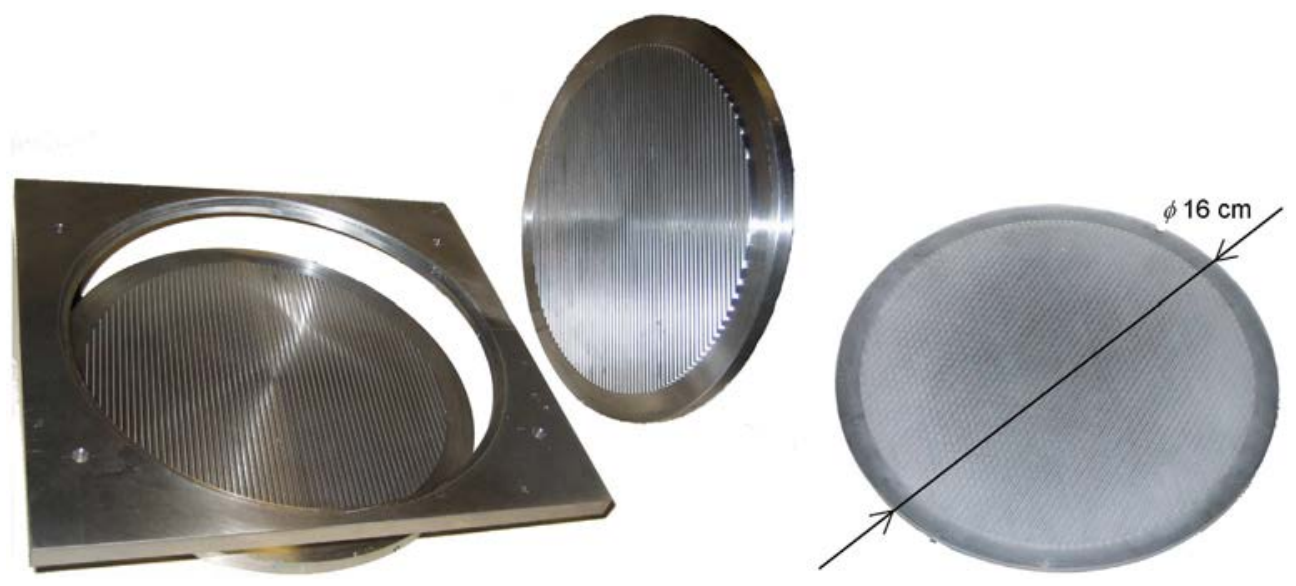

Fig. 3. Photograph of the mould used to process the crenellated membranes (left) and photograph of a resulting crenellated plate (right).

\section{Experimental results}

\subsection{Mechanical behaviour of the silicone rubber}

Results obtained during the uniaxial, planar and equibiaxial tension tests are summarised in Fig. 4. In comparison with the results presented in Meunier et al. (2008), curves show first that the studied silicone rubber is stiffer; second the strain-hardening appears earlier, as expected from its longer curing time (Rey et al., 2013), but the behaviour remains hyperelastic. Thus, a classical and simple hyperelastic constitutive equation is used to fit the experimental data, i.e. the (Yeoh, 1990) model, the strain energy function is defined by:

$W=C_{10}\left(I_{1}-3\right)+C_{20}\left(I_{1}-3\right)^{2}+C_{30}\left(I_{1}-3\right)^{3}$.

In the above equations, $C_{10}, C_{20}, C_{30}$ are material parameters, and $I_{1}=\operatorname{tr}(\mathbf{C})$ is the first invariant of the right Cauchy-Green strain tensor $\mathbf{C}$ where $\mathbf{C}=\mathbf{F}^{t} \mathbf{F}$ and $\mathbf{F}$ is the deformation gradient. From the knowledge of the initial thickness $t_{0}$ of the tested homogeneous flat silicone membranes, the tensile elongation $\lambda$, the

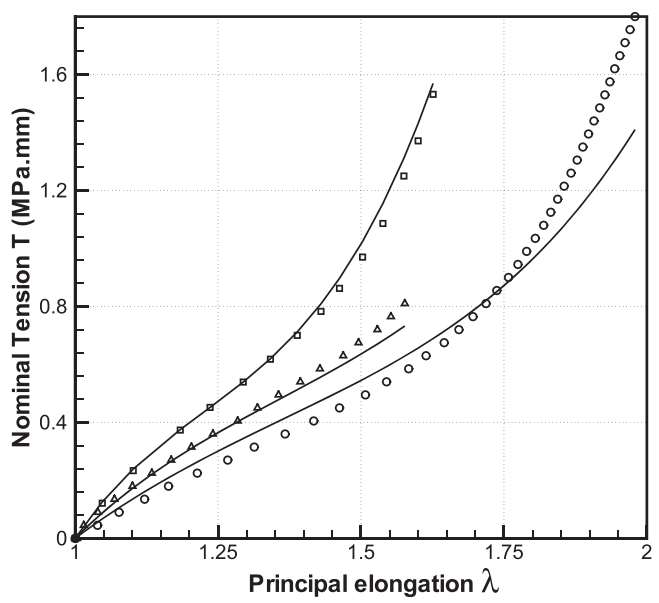

Fig. 4. Principal tension - principal elongation curves obtained with flat and homogeneous silicone rubber membranes processed in this work. The experimental data of uniaxial tensile test $(\bigcirc)$, pure shear test $(\Delta)$ and equibiaxial tension $(\square)$ are compared with the predictions of Yeoh models $(-)$. tension $T$ in the membranes is uniaxial and its only component can be estimated as:

$T=2 t_{0}\left(\lambda-\frac{1}{\lambda^{2}}\right) \frac{\partial W}{\partial I_{1}}$

This allowed to fit the model parameters. Their values are $C_{10}=0.246 \mathrm{MPa}, C_{20}=-4.28 \cdot 10^{-3} \mathrm{MPa}$ and $C_{30}=0.0160 \mathrm{MPa}$. The model predictions are plotted in Fig. 4. The Yeoh model captures correctly the trends exhibited by the silicone rubber whatever the investigated loading path, with only three material parameters. Consequently, the Yeoh model was used in the following as a relevant model for this silicone rubber.

\subsection{Mechanical behaviour of the architectured membranes}

First, due to the equilibrated mesostructure of the specimen, no out-of-plane curl of the membranes was observed during the tensile tests. The results of the tensile tests for the different orientation of the crenels are presented in Fig. 5. Fig. 5(a) presents the evolution of the global transversal elongation as function of the longitudinal elongation, by means of DIC measures. The continuous line corresponds to a homogeneous incompressible membrane. Most of the crenellated membranes behave as homogeneous incompressible membrane except for angles $2 \alpha=135^{\circ}$ and $155^{\circ}$. These membranes present less in-plane shrinkage than the others ones.

Fig. 5(b) illustrates the evolution of the nominal tension $T$ with the tensile elongation $\lambda$. It emphasises the strong influence of the relative orientation of the crenels on the membrane mechanical behaviour: the lower value of $2 \alpha$ (i.e. the more the crenels were orientated along the tensile direction) requires higher tension to deform the membranes. Also notice that the experimental curves are bounded by two continuous lines. These bounds were obtained from simple analytical estimations, deduced from the knowledge of the mechanics of the silicone rubber (see previous subsection). They represent the tensile curves obtained with homogeneous flat membranes of different thickness $t_{e q}$. The lowest is obtained for $t_{e q}=t_{0}$ which corresponds to a membrane without crenel (i.e. with $t_{c}=0 \mathrm{~mm}$ ). This bound should be very close to the behaviour obtained with a crenellated membrane with $2 \alpha=180^{\circ}$. The highest bound is such that $t_{e q}=t_{0}+2 t_{c} w_{1} /\left(w_{1}+w_{2}\right)$ which corresponds to a crenellated specimen with $2 \alpha=0^{\circ}$. Hence, this graph clearly highlights the possible range of available mechanical behaviours that can be obtained from the studied architectured membranes, simply by tuning the angle $2 \alpha$. 

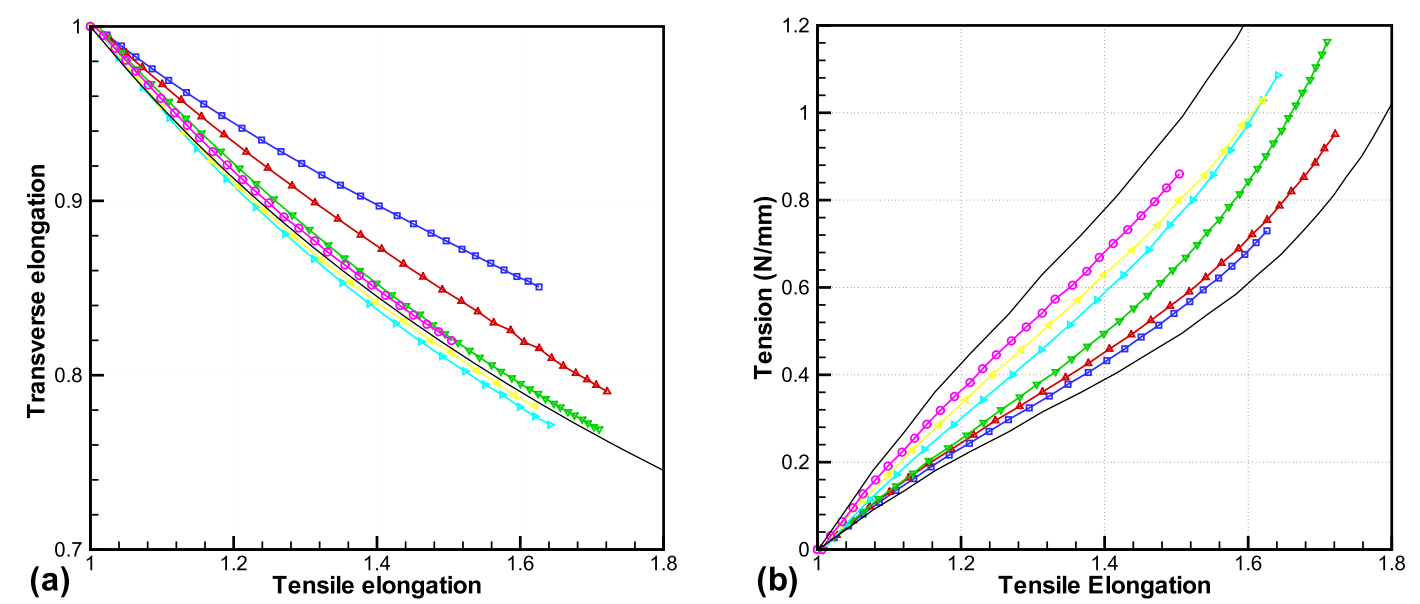

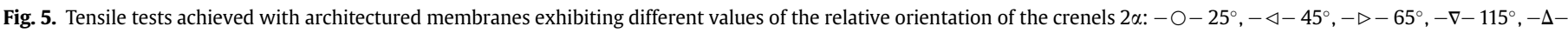

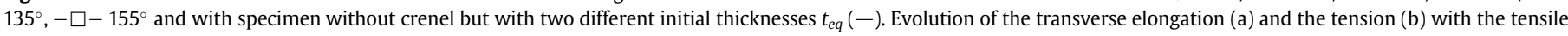
elongation.

\section{Analytical macroscopic model}

The topic of this part is to propose simple constitutive equations able to describe the equivalent behaviour of the crenellated membranes.

The proposed macroscopic model is based on simple considerations. It is considered that the mechanical behaviour of the membrane can be modelled as the sum of the mechanical behaviour of the different parts of the membrane i.e. the flat membrane in the middle of the specimen and the crenels. It is also assumed that there is no interaction between the mechanical behaviour of the homogeneous membrane and the crenels, but also between the two crenels. First an isotropic incompressible homogeneous membrane of constant initial thickness $t_{0}$ is used to model the flat membrane, and second a tension-compression behaviour of the incompressible slender crenels is considered. The crenels are initially oriented in a unit direction $\mathbf{A}_{i}(i=1,2)$, after transformation, their orientations are given by unit direction $\mathbf{a}_{i}$. Thus, the tension in the studied membranes is supposed to be written as:

$\tilde{\mathbf{T}}=\tilde{\mathbf{T}}^{m}+\tilde{\mathbf{T}}^{c}$

where $\tilde{\mathbf{T}}^{m}$ stands for the contribution of the flat membrane, and where $\tilde{\mathbf{T}}^{c}$ is that induced by the two stretched crenels. The notation "w stands for second-order tensors ${ }^{2}$ (If there is no crenels, in uniaxial extension, the tension can be written as in Equation (2)).

As presented in paragraph 3.1, the Yeoh model is adapted to represent the silicone rubber. Thus, it is used to describe both the membrane and the crenels. The tension in the membrane is thus simply written as:

$\left.\tilde{\mathbf{T}}^{m}=2 t_{0} \frac{\partial W}{\partial I_{1}} \quad \tilde{\mathbf{B}}-\frac{1}{\tilde{J}^{2}} \tilde{\mathbf{I}}\right) \tilde{\mathbf{F}}^{-t}$

with $\tilde{\mathbf{I}}$ the identity tensor, $\tilde{\mathbf{B}}=\tilde{\mathbf{F}} \tilde{\mathbf{F}}^{t}$ is left Cauchy-Green tensor, $\tilde{J}^{2}=\operatorname{det} \tilde{\mathbf{B}}$, thus $I_{1}=\tilde{I}_{1}+1 / \tilde{J}^{2}$ (details are given in Appendix A), and where:

\footnotetext{
2 Please notice that in the following such a notation will be used for vectors but also tensors. For vectors, the third component is zero, and for tensors, the components of which along the $\mathbf{e}_{\mathrm{i}} \otimes \mathbf{e}_{3}$ and $\mathbf{e}_{3} \otimes \mathbf{e}_{\mathrm{i}}(i=1,2,3)$ are zeros.
}

$$
\left.\left.\frac{\partial W}{\partial I_{1}}=C_{10}+2 C_{20} \quad \tilde{I}_{1}+\frac{1}{\tilde{J}^{2}}-3\right)+3 C_{30} \tilde{I}_{1}+\frac{1}{\tilde{J}^{2}}-3\right)^{2}
$$

The crenels are considered to present a mechanical response only in tension-compression. The elongation $\lambda^{c}$ of each crenel $(i=1,2)$ is simply defined by means of anisotropic invariant which are linked to the elongation (Spencer, 1971) $\lambda_{i}^{c 2}=\tilde{I}_{i}^{4}=\mathbf{A}_{i} \tilde{\mathbf{C}} \mathbf{A}_{i}$. The mechanical properties of the two crenels are identical. Thus the tension in the crenels can be written as:

$\tilde{\mathbf{T}}^{c}=2 f_{c} W_{c}\left(\mathbf{a}_{1} \otimes \mathbf{a}_{1}+\mathbf{a}_{2} \otimes \mathbf{a}_{2}\right) \tilde{\mathbf{F}}^{-t}$

where $f_{c}$ is the fraction of bar along the membranes (It corresponds to the ratio of the volume of crenels compared to the volume of the flat membrane) and $W_{c}$ takes into account the strain energy and the uniaxial loading state. It can be determined simply by using a hyperelastic energy in a uniaxial tension loading state. According to geometry specifications of the crenels as illustrated in Fig. 1, $f_{c}=t_{c} w_{1} /\left(w_{1}+w_{2}\right)$, and in case of the Yeoh model:

$$
\begin{aligned}
W_{c}= & \left(\lambda_{i}^{c 2}-\frac{1}{\lambda_{i}^{c}}\right)\left(C_{10}+2 C_{20}\left(\lambda_{i}^{c 2}+\frac{2}{\lambda_{i}^{c}}-3\right)\right. \\
& \left.+3 C_{30}\left(\lambda_{i}^{c 2}+\frac{2}{\lambda_{i}^{c}}-3\right)^{2}\right)
\end{aligned}
$$

Consequently, from the knowledge of the constitutive parameters of the Yeoh model $C_{i 0}$, the initial geometry of the crenels, the initial relative angle between the crenels $2 \alpha$, the tension contribution $\tilde{\mathbf{T}}^{c}$ can be estimated analytically, as well as the tension in the membrane $\tilde{\mathbf{T}}^{m}$ for any macroscopic transformation gradient $\tilde{\mathbf{F}}$.

\section{Multi-scale modelling}

The mechanics of a heterogeneous and hyperelastic membrane subjected to an in-plane mechanical loading identical to the above experiments is here studied and upscaled. From the knowledge of the mechanical loading of the membrane, its heterogeneous geometry and the mechanical behaviour of its bulk material, the aim is to obtain a set of homogenised balance and constitutive equations describing the mechanical behaviour of the considered 
membrane at the macroscale, together with suitable boundary value problems to be solved on Representative Elementary Volumes in order to estimate the effective properties of the homogenised architectured membrane. To do so, the homogenisation with multiple scale asymptotic expansions is used: the theoretical developments presented herein are similar to those proposed by Pruchnicki (1998a, b) for membranes made up of Neo-Hookean (Treloar, 1943) and Saint Venant-Kirchhoff materials, his work is revisited in order to extend it to any strain energy density.

\subsection{Problem statement}

\subsubsection{Geometry of the membrane at the microscale}

The deformation of a thin heterogeneous (here architectured) plate, e.g. that shown in Fig. 6(a) in its initial and undeformed configuration $\mathcal{C}_{0}$, with an initial average thickness $t_{0}^{m}$ along the $\mathbf{e}_{3}$ direction and with a characteristic in-plane initial macroscopic dimension $L_{0}$ in $\left(\mathbf{e}_{1}, \mathbf{e}_{2}\right)$. Each material point $M$ of the plate is located by its initial spatial position $\mathbf{X}$. Upon a given mechanical loading (see below), the plate is deformed into a current configuration $\mathcal{C}$, and the spatial positions of its material points are noted $\mathbf{x}$. The displacement field $\mathbf{u}$ of the material point $M$ together with the deformation gradient $\mathbf{F}$ from $\mathcal{C}_{0}$ to $\mathcal{C}$ are respectively defined as:

$\mathbf{u}(\mathbf{X})=\mathbf{X}-\mathbf{X}$ and $\mathbf{F}=\operatorname{Grad}_{X} \mathbf{X}=\mathbf{I}+\operatorname{Grad}_{X} \mathbf{u}$,

where $\operatorname{Grad}_{X}$ represents the gradient of $\mathbf{x}$ or $\mathbf{u}$ with respect to the initial configuration and to the position vector $\mathbf{X}$. The considered plate is made up of a periodic in plane repetition of a REV occupying a domain $\Omega_{R E V}$ of initial and current volumes $V_{0}$ and $V$, of external surfaces $\partial \Omega_{R E V}$. Such a two-dimension periodicity is here characterised by two periodicity vectors, $\mathbf{P}_{1}$ and $\mathbf{P}_{2}$ belonging to $\left(\mathbf{e}_{1}, \mathbf{e}_{2}\right)$ and represented in Fig. 6. The characteristic in-plane initial dimension of this REV is $l_{0}$, and its initial in-plane area $S_{0}$ can be expressed as $S_{0}=\left\|\mathbf{P}_{1} \times \mathbf{P}_{2}\right\|$. It is further supposed that $l_{0}$ and the average thickness $e_{0}$ of the REV are of the same order of magnitude, i.e. $e_{0} / l_{0} \approx \mathcal{O}(1)$, and that they are very small with respect to the inplane size $L_{0}$ of the plate, i.e. $e_{0} / L_{0} \approx l_{0} / L_{0}=\varepsilon$, where the scale separation parameter $\varepsilon$ represents the dimension of the heterogeneities to the length of the whole structure is very small (Pruchnicki, 1998a, b). A typical example of such REV's in its initial configuration $\mathcal{C}_{0}$ is shown in Fig. $6(\mathrm{~b})$.

\subsubsection{Mechanical loading of the membrane at the microscale}

For a sake of simplicity, it is assumed that the plate is only subjected to a quasi-static and in-plane mechanical loading without external volumetric force and couple. This mechanical loading transforms the plate from $\mathcal{C}_{0}$ to $\mathcal{C}$, the consequences on the REV are detailed:
1. the external surfaces $\Gamma_{e}$ of the REV, i.e. all the surfaces of the REV which are not in connection with the REV's neighbours, are not loaded. Hence, by noting respectively $\pi$ and $\mathbf{N}_{e}$ the first Piola-Kirchhoff stress tensor of the membrane and the local external unit vector normal to $\Gamma_{e}$ in $\mathcal{C}_{0}$, the surfaces $\Gamma_{e}$ are subjected to the following boundary condition:

$\pi \mathbf{N}_{e}=0$ on $\Gamma_{e}$

2. The surfaces of the REV which are in connection with the REV's neighbours (i.e. the lateral surfaces $\Gamma_{p}$ of the REV) are subjected to periodic boundary conditions. This means that the continuity of the displacement and stress fields must be imposed. Hence, for each material point belonging to a given surface $\Gamma_{p}$ of local external unit normal $\mathbf{N}_{p}$, the following conditions are imposed:

$$
\left.\begin{array}{l}
\mathbf{u}(\mathbf{X})=\mathbf{u}\left(\mathbf{X}_{ \pm} \mathbf{P}_{i}\right) \\
\left(\pi(\mathbf{X})-\pi\left(\mathbf{X}_{ \pm} \mathbf{P}_{i}\right)\right) \mathbf{N}_{p}=0
\end{array}\right\} \quad \text { on } \Gamma_{p}
$$

3. the first momentum balance equation corresponding to this problem and to be solved within the membrane is:

$$
\operatorname{Div}_{X} \pi=0
$$

where $\mathbf{D i v}_{X}$ is the divergence operator with respect to $\mathcal{C}_{0}$ and position vectors $\mathbf{X}$.

4. the second momentum balance equation imposes the first Piola-Kirchhoff stress tensor $\pi$ the symmetry condition: $\pi \mathbf{F}^{t}=\mathbf{F} \pi^{t}$.

\subsubsection{Mechanical behaviour of the constituent of the membrane}

The plate is supposed to be made up of a homogeneous hyperelastic material, so the stress tensor $\pi$ can be written as $\pi=\partial W(\mathbf{F}) / \partial \mathbf{F}$ where $W$ represents the local strain energy function per unit of undeformed volume. $W$ must be an objective quantity and can be written, as a function of the Cauchy Green strain tensor C. For the rest of the document, $W$ is also subjected to two additional restrictions which are listed below:

1. $W$ is also sufficiently smooth to admit a Taylor expansion around the neighbouring $\mathbf{F}+\delta \mathbf{F}$ of $\mathbf{F}$ and to allow the stress tensor $\pi$ to be also expressed as a Taylor expansion too:

$\pi(\mathbf{F}+\delta \mathbf{F})=\pi(\mathbf{F})+\frac{\partial \pi}{\partial \mathbf{F}}: \delta \mathbf{F}+\ldots$

2. The whole REV is stress-free in $\mathcal{C}_{0}$. It is supposed that this initial stress-free state corresponds to a minimum of $W: \forall \mathbf{F} \neq \mathbf{I}, W(\mathbf{F})$
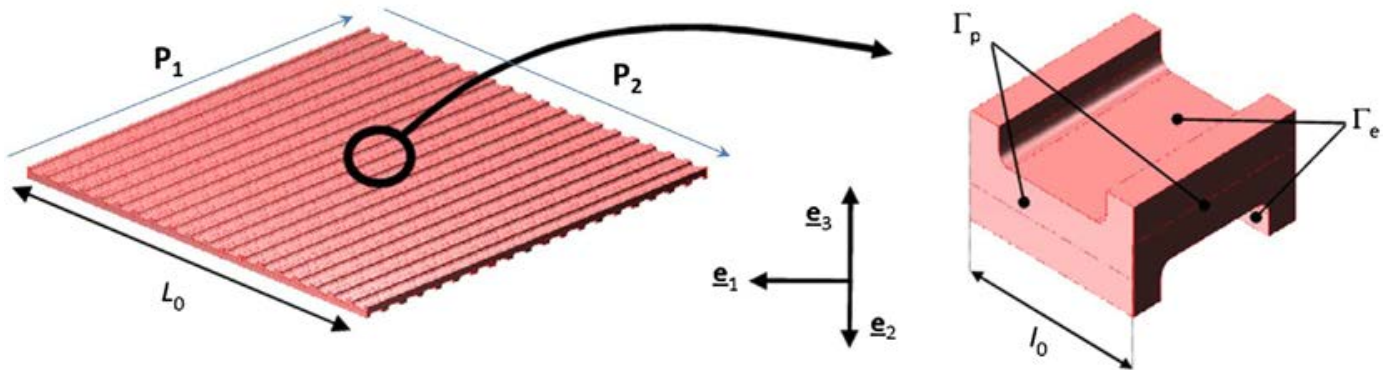

Fig. 6. (a) Scheme of a typical heterogeneous plate under consideration, (b) corresponding periodic REV. 
(a)

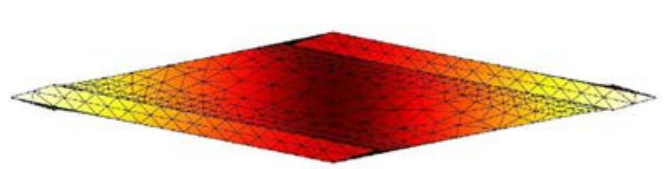

(c)

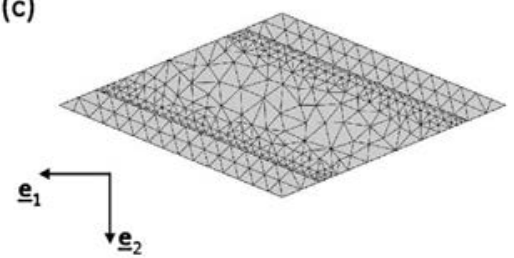

(b)

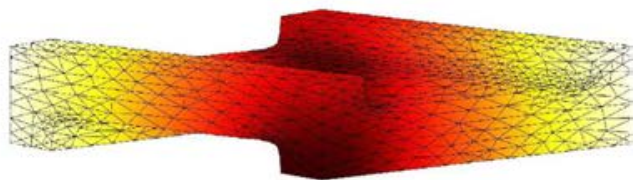

(d)

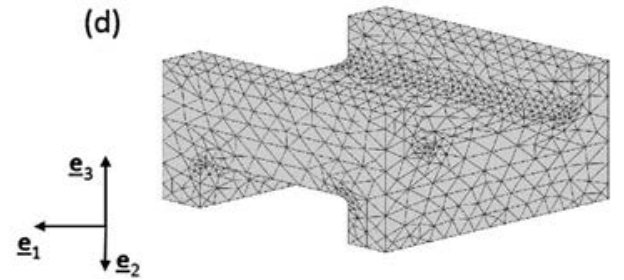

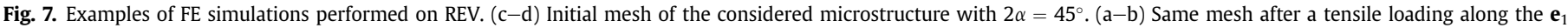

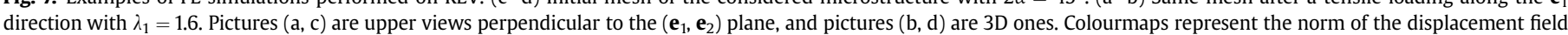
from 0 (darker colour) to its maximal value. (For interpretation of the references to colour in this figure legend, the reader is referred to the web version of this article.)

$>W(\mathbf{I})$. Thus, the strain energy function of the REV per unit of initial surface, $\mathcal{W}$, corresponding to a deformed configuration $\mathcal{C}$ and defined as

$\mathcal{W}(\mathcal{C})=\frac{1}{S_{0}} \int_{\Omega_{R E V}} W(\mathbf{F}) \mathrm{d} V_{0}$

admits a minimum in the initial configuration: $\forall \mathcal{C} \neq \mathcal{C}_{0}$, $\mathcal{W}(\mathcal{C})>\mathcal{W}\left(\mathcal{C}_{0}\right)$.

\subsection{Theoretical upscaling}

In order to obtain the equivalent macroscopic mechanical behaviour of the as-described local membrane problem, the homogenisation method with multiple scale asymptotic expansions is now used (Bensoussan et al., 1978; Sanchez-Palencia, 1980; Pruchnicki, 1998a, b).

\subsubsection{Separated spaces variables}

Provided a good scale separation between $L_{0}$ and $l_{0}$ (and $e_{0}$ ), the above problem can be tackled by introducing two distinct and independent space variables:

(i) a macroscopic variable: $\tilde{\mathbf{X}}=\tilde{X}_{\alpha} \mathbf{e}_{\alpha}, \quad \alpha=1,2$

(ii) a microscopic variable: $\mathbf{Y}=\mathbf{X} / \varepsilon$.

The body has thus two length scales, a global length scale that is described by the macroscopic in-plane space variable $\tilde{\mathbf{X}}$ and a local length scale described by the microscopic one $\mathbf{Y}$ which serves to describe the fluctuations of these fields within the REV. Thus, provided a good scale separation, any scalar function $\varphi(\mathbf{X})$ can be written as a function of $\tilde{\mathbf{X}}$ and $\mathbf{Y}$, i.e. $\varphi(\tilde{\mathbf{X}}, \mathbf{Y})$. Then, the differential operators are also calculated with respect to $\tilde{\mathbf{X}}$ and $\mathbf{Y}$. For example, the gradient of the scalar field $\varphi$ becomes:

$$
\operatorname{Grad}_{X} \varphi \rightarrow \varepsilon^{-1} \operatorname{Grad}_{Y} \varphi+\widetilde{\operatorname{Grad}_{\tilde{X}} \varphi}
$$

\subsubsection{Asymptotic expansions}

It is further assumed that the displacement field $\mathbf{u}(\tilde{\mathbf{X}}, \mathbf{Y})$ can be expressed as an asymptotic expansion in powers of $\varepsilon$ :
$\mathbf{u}=\mathbf{u}^{(0)}(\tilde{\mathbf{X}}, \mathbf{Y})+\varepsilon \mathbf{u}^{(1)}(\tilde{\mathbf{X}}, \mathbf{Y})+\varepsilon^{2} \mathbf{u}^{(2)}(\tilde{\mathbf{X}}, \mathbf{Y})+\ldots$

where the displacement fields $\mathbf{u}^{(i)}$ are supposed to be of the same order of magnitude and Y-periodic on the lateral surfaces $\Gamma_{p}$ of the REV. The order of magnitude difference between the terms is only due to the factor $\varepsilon$ which is small. Hence, the gradient of the displacement field can be written as:

$$
\operatorname{Grad}_{X} \mathbf{u}=\varepsilon^{-1} \operatorname{Grad}^{(-1)} \mathbf{u}+\operatorname{Grad}^{(0)} \mathbf{u}+\varepsilon \operatorname{Grad}^{(1)} \mathbf{u}+\ldots
$$

by noting $\left\{\begin{array}{l}\operatorname{Grad}^{(-1)} \mathbf{u}=\operatorname{Grad}_{Y} \mathbf{u}^{(0)} \\ \operatorname{Grad}^{(0)} \mathbf{u}=\operatorname{Grad}_{Y} \mathbf{u}^{(1)}+\widetilde{\operatorname{Grad}_{\tilde{X}}} \mathbf{u}^{(0)} \\ \operatorname{Grad}^{(1)} \mathbf{u}=\operatorname{Grad}_{Y} \mathbf{u}^{(2)}+\widetilde{\operatorname{Grad}_{\tilde{X}}} \mathbf{u}^{(1)} \\ \ldots\end{array}\right.$

Similarly, the deformation gradient becomes:

$\mathbf{F}=\varepsilon^{-1} \mathbf{F}^{(-1)}+\mathbf{F}^{(0)}+\varepsilon \mathbf{F}^{(1)}+\ldots$

by noting $\left\{\begin{array}{l}\mathbf{F}^{(-1)}=\mathbf{G r a d}^{(-1)} \mathbf{u} \\ \mathbf{F}^{(0)}=\mathbf{I}+\mathbf{G r a d}^{(0)} \mathbf{u} \\ \mathbf{F}^{(1)}=\operatorname{Grad}^{(1)} \mathbf{u} \\ \ldots\end{array}\right.$.

Therefrom, the homogenisation method consists (i) in introducing the above asymptotic expansion in the problem (9-11) and (ii) in identifying and solving the problems arising at the different $\varepsilon$-orders Pruchnicki (1998a, b). Below, the main results of this upscaling process are briefly summarised, some of the details of the theoretical developments being given in the appendixes:

- From the assumptions stated for $W$, it can be shown (see appendix B) that the first order displacement field $\mathbf{u}^{(0)}(\tilde{\mathbf{X}}, \mathbf{Y})$ only depends on the in-plane macroscopic space variable, i.e. $\mathbf{u}^{(0)}=\mathbf{u}^{(0)}(\tilde{\mathbf{X}})$. $\mathbf{u}^{(0)}$ represents the macroscopic displacement field and will be further noted $\tilde{\mathbf{U}}$.

- The macroscopic equivalent continuum is a 2D membrane contained in the $\left(\mathbf{e}_{1}, \mathbf{e}_{2}\right)$ plane. Its first momentum balance equation in the initial configuration is expressed as (see Appendix D): $\widetilde{\operatorname{Div}}_{\tilde{X}} \tilde{\mathbf{T}}=\tilde{0}$ where the macroscopic in-plane tension $\tilde{\mathbf{T}}$ is defined as: 
$\tilde{\mathbf{T}}=\frac{1}{S_{0}} \int_{U_{R E V}} \frac{\partial W\left(\mathbf{F}^{(0)}\right)}{\partial \mathbf{F}^{(0)}} \mathrm{d} V_{0}$,

and is subjected to the symmetry condition: $\tilde{\mathbf{T}} \tilde{\mathbf{F}}^{t}=\tilde{\mathbf{F}} \tilde{\mathbf{T}}^{t}$ where $\tilde{\mathbf{F}}$ represents the macroscopic in-plane transformation gradient:

$$
\tilde{\mathbf{F}}=\tilde{\mathbf{I}}+\widetilde{\operatorname{Grad}_{\tilde{X}}} \tilde{\mathbf{U}}
$$
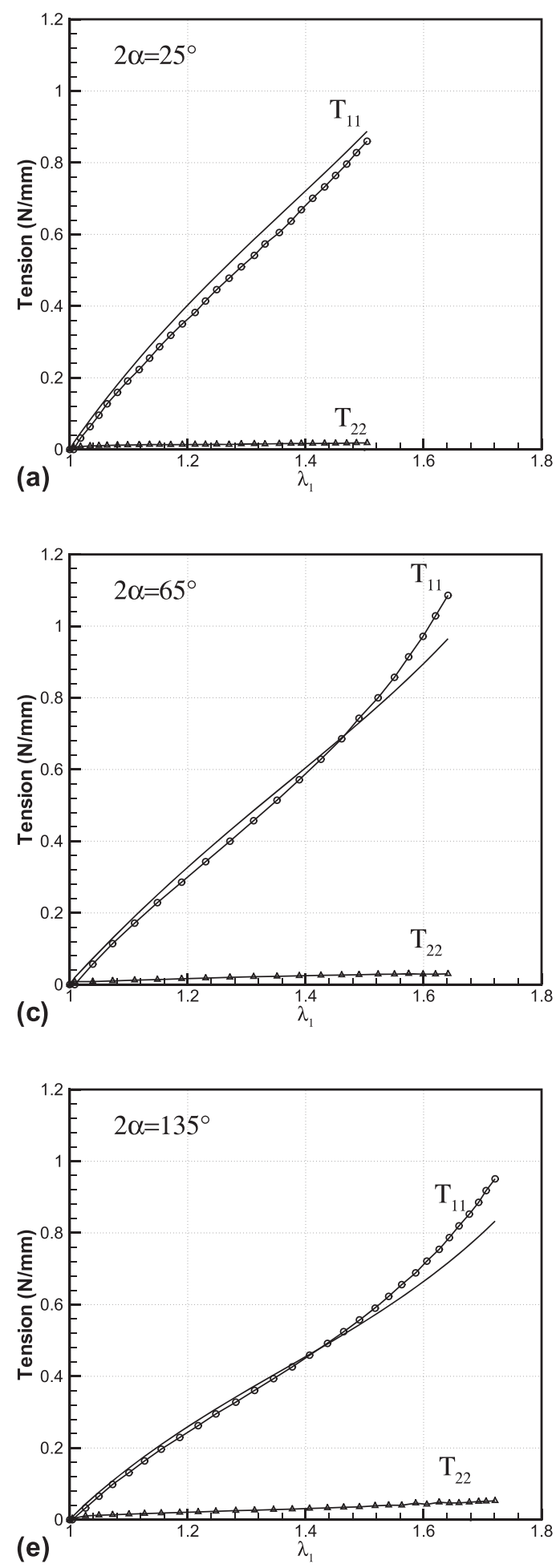

- The macroscopic in-plane behaviour of the membrane is hyperelastic (see Appendix E). It can be defined from the knowledge of the macroscopic in-plane strain energy function per unit of initial thickness $\tilde{\mathcal{W}}$, which depends on both the microstructure of the REV and the macroscopic in-plane transformation gradient $\tilde{\mathbf{F}}$. Hence, the macroscopic in-plane tension is expressed as:

$\tilde{\mathbf{T}}=\frac{\partial \tilde{\mathcal{W}}}{\partial \tilde{\mathbf{F}}}$
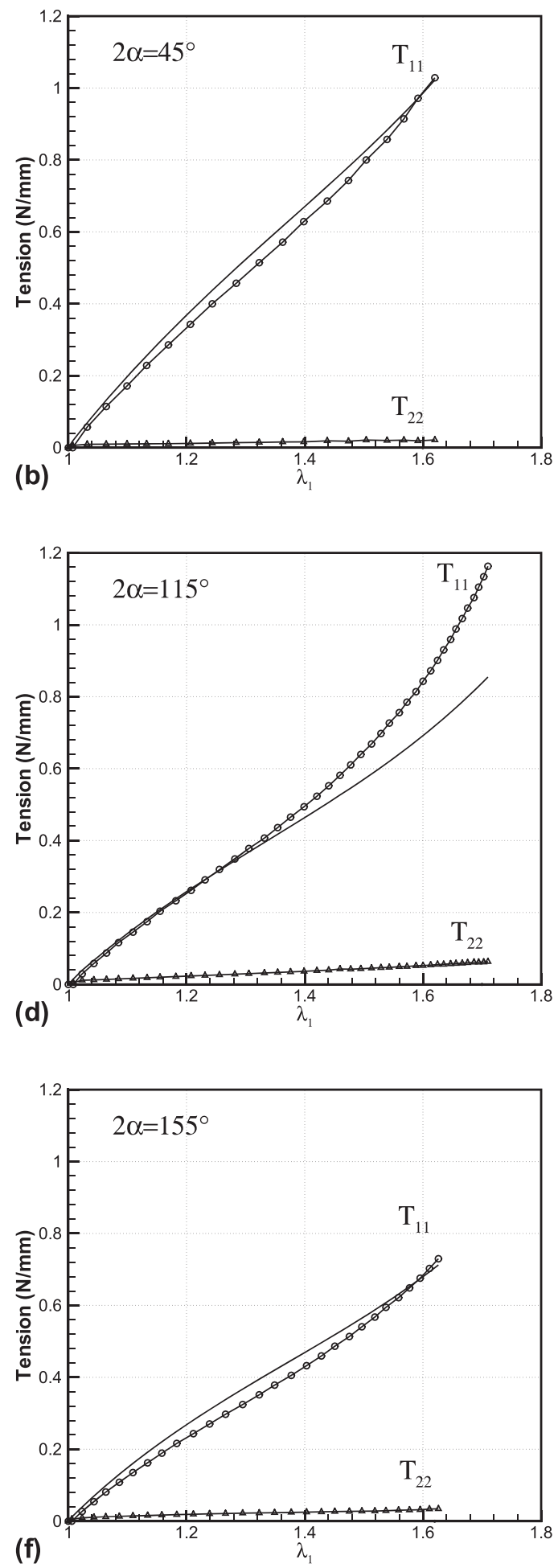

Fig. 8. Comparison between the proposed analytical model $\left((-)\right.$ for $T_{11}$ and $-\Delta-$ for $\left.T_{22}\right)$ and the experimental results $-O-$ with the six relative orientations of crenels $2 \alpha$. 
- The quantitative determination of this macroscopic constitutive equation can be handled experimentally, or analytically/ numerically. For the last case, the following route can be taken:

1. Choose a relevant set of macroscopic in-plane transformation gradients $\tilde{\mathbf{F}}$

2. Solve the self-equilibrium of the REV for each of them (see Appendix C):

$$
\left\{\begin{array}{l}
\operatorname{Div}_{Y} \pi^{(0)}=0 \\
\pi^{(0)}=\frac{\partial W\left(\mathbf{F}^{(0)}\right)}{\partial \mathbf{F}^{(0)}} \\
\mathbf{F}^{(0)}=\tilde{\mathbf{F}}+\mathbf{e}_{3} \otimes \mathbf{e}_{3}+\mathbf{G r a d}_{Y} \mathbf{u}^{(1)} \\
\pi^{(0)} \mathbf{N}_{e}=0
\end{array} \quad \text { in } \Omega_{R E V}\right.
$$

In the last boundary value problem, the macroscopic in-plane transformation gradient $\tilde{\mathbf{F}}$ is given and constant in the REV, and the unknown displacement field to be determined, $\mathbf{u}^{(1)}$, is periodic.

3. Calculate the macroscopic in-plane tension $\tilde{\mathbf{T}}$ according to (20).

4. The formulation is valid for any strain energy density $\tilde{\mathcal{W}}(\tilde{\mathbf{F}})$. As a consequence, the one that fits the experimental data of the material must be chosen.
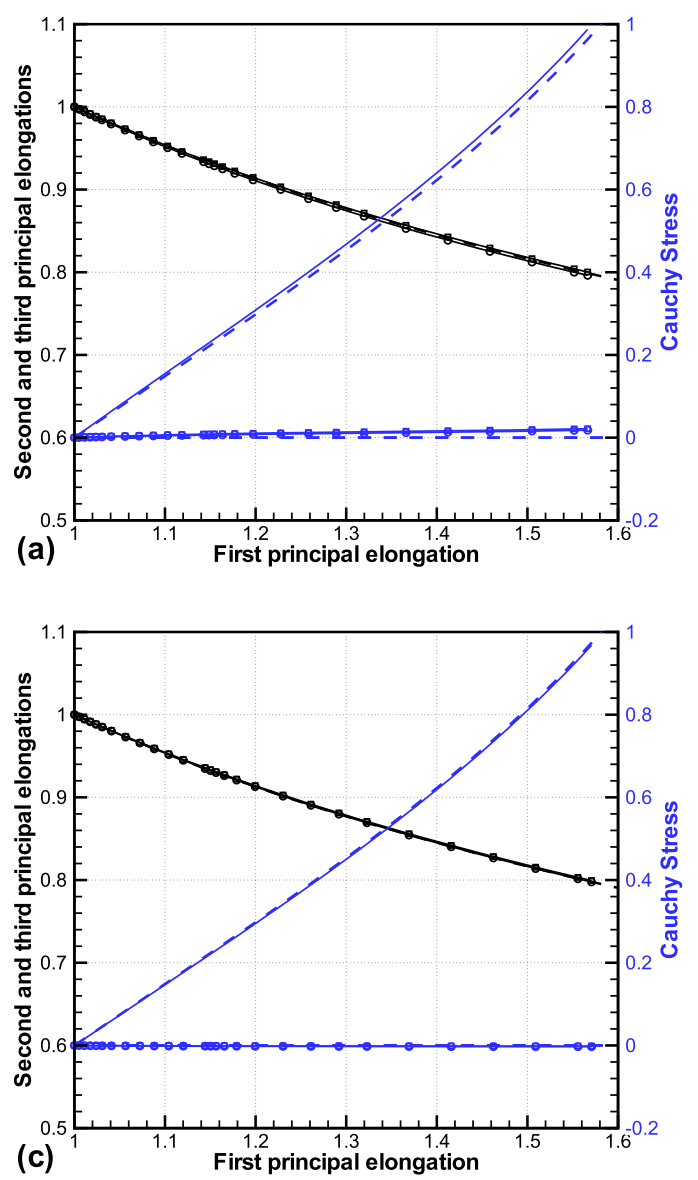

\subsection{Application to the tested silicone membranes}

In this section, the effective properties of the tested hyperelastic silicone membranes are computed by following the previous theoretical framework. These numerical estimations are then compared with the experimental results. For that purpose, the localisation problem (Equation (23)) was solved on REVs of all the tested membranes. As an example, the REV corresponding to an angle $2 \alpha=45^{\circ}$ is presented in Fig. 7 .

To realise the simulations, the weak formulation of (Equation (23)) was implemented in the Finite Element software Comsol 3.5 with a quasi-incompressible formulation. The REVs were meshed with quadratic elements (see the example in Fig. 7). The mechanical behaviour of the silicone was modelled by using the Yeoh model. Besides, to model the tensile tests performed in Section 3.2, each REV was subjected to in-plane transformation gradients $\tilde{\mathbf{F}}$ which exactly corresponded to those measured at the macroscale with the DIC method. An example of a deformed REV is presented in Fig. 7.

\section{Discussion}

In the previous sections, two models were proposed, an analytical one and a REV finite element models one. The main difference between the two models is that finite element calculation is time consuming and is not adapted to a rapid optimisation. Nevertheless, the abilities of the two models to represent experimental results are studied.
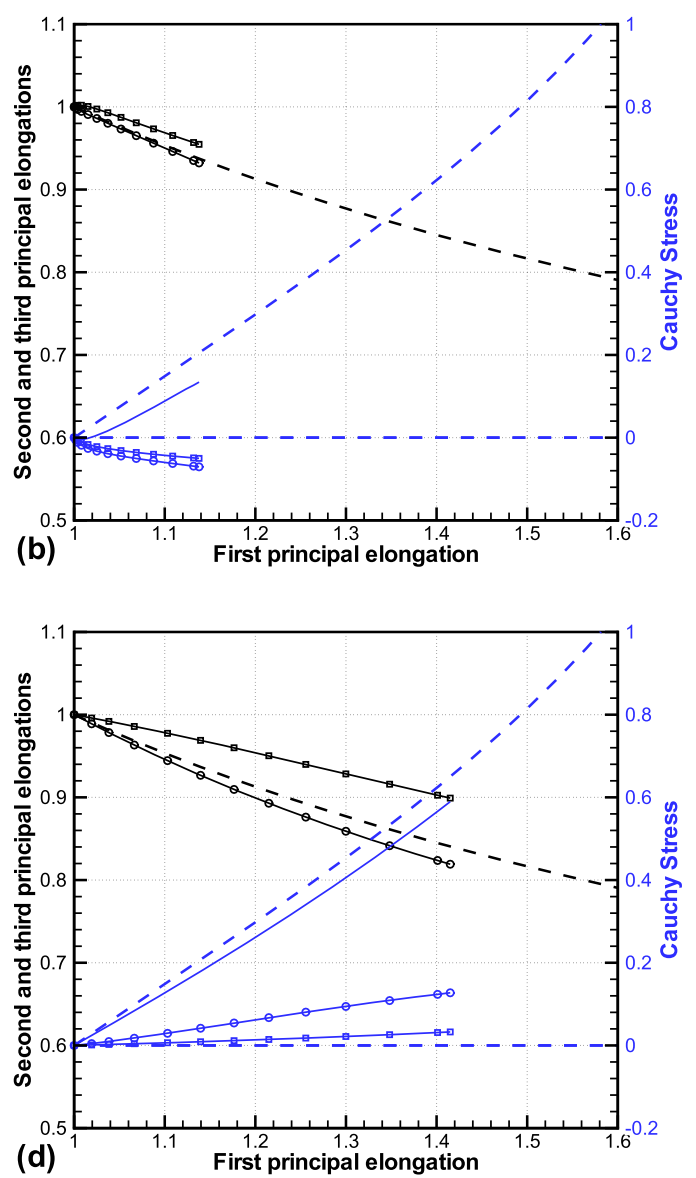

(d)

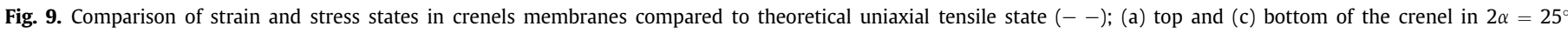
membranes; (b) top and (d) bottom of the crenel in $2 \alpha=115^{\circ}$ membranes. 


\subsection{Abilities of the analytical model}

Tension-elongation curves $\tilde{T}_{11}-\lambda_{1}$ and $\tilde{T}_{22}-\lambda_{1}$ plotted in Fig. 8 give the comparison between the predictions given by the analytical model and the experimental results.

For the two tension components $\left(\tilde{T}_{11}\right.$ and $\left.\tilde{T}_{22}\right)$, this figure shows that trends given by the proposed analytical model, rather fit well the simulation results. A slight deviation occurs for large angles $2 \alpha$.
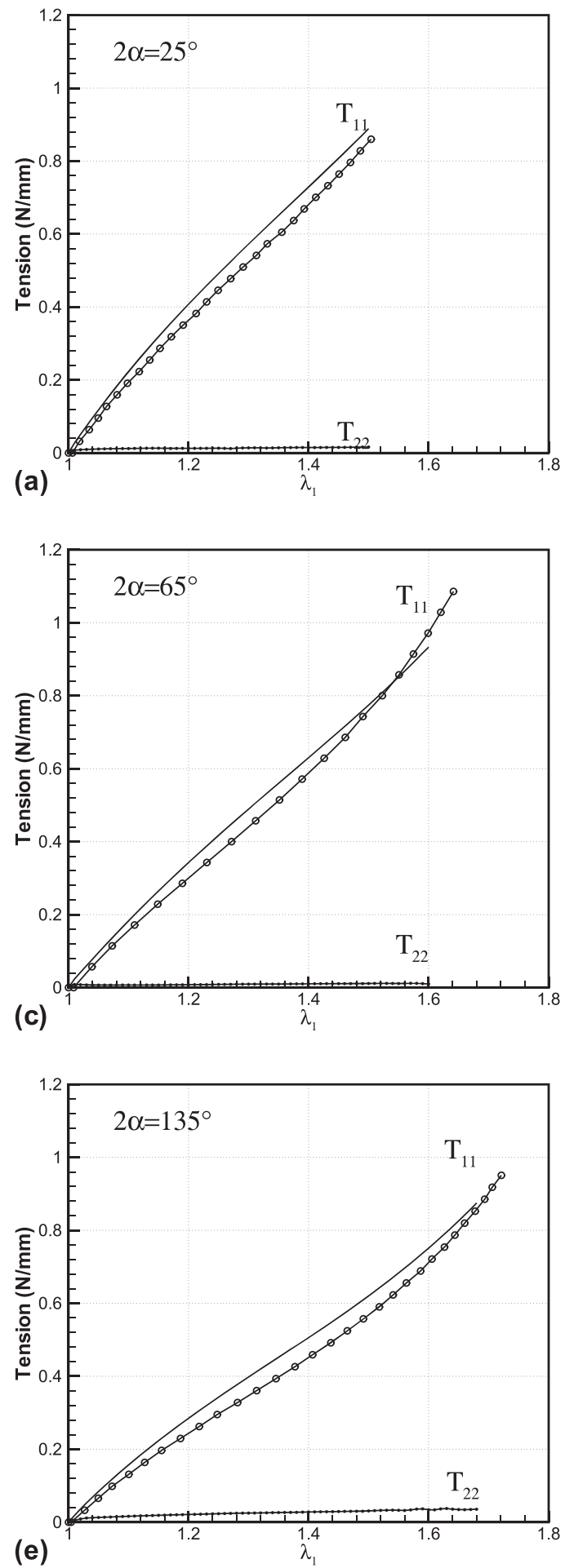

These results are quite good for a model that does not necessitate any additional parameter than those of the bulk material.

The main reason that can explain the deviation between the analytical model and the numerical simulations for the largest angles is the loading state in the crenels. In the analytical model, there are supposed to be in uniaxial extension, that means that the two transversal elongations must be equal and the stress state must be uniaxial. Fig. 9 illustrates the strain and stress states in the
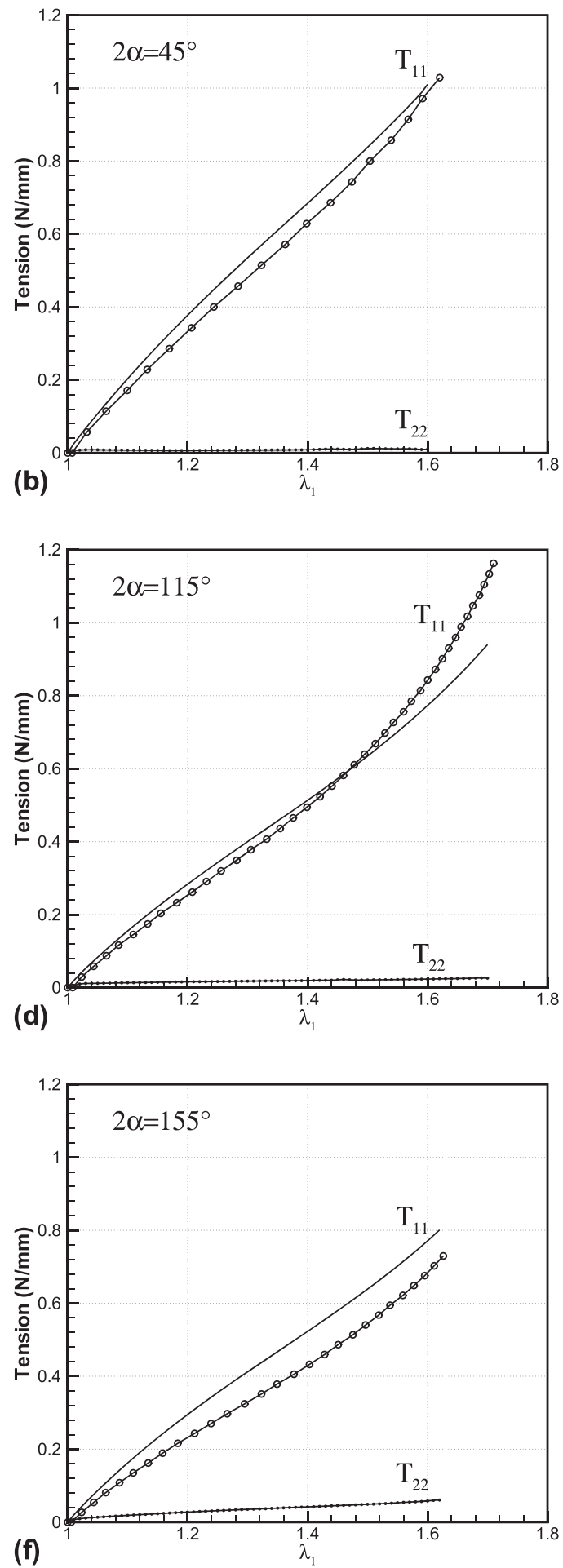

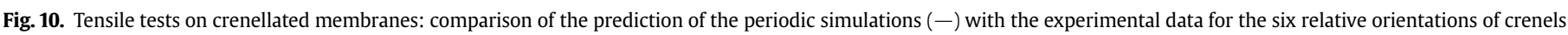
$(-\bigcirc-)$. 
crenels of the membrane, by means of the finite element simulation. The second and third principal elongations are plotted versus the maximal principal elongation, under incompressible hypothesis, if $\lambda$ is the tension elongation, the transverse elongations are $1 / \sqrt{\lambda}$ in uniaxial extension. In a same way, the three principal Cauchy stress are represented. They are compared to the theoretical uniaxial tensile stress. Only results for the angles $2 \alpha=25^{\circ}$ and $2 \alpha=115^{\circ}$ are represented, two figures are plotted for each angle, one that corresponds to the top of the crenel and one to the bottom. For angles of $2 \alpha=45^{\circ}$ and $2 \alpha=65^{\circ}$, the results (not presented here for a sake of clarity) are similar to $2 \alpha=25^{\circ}$. It clearly appears that the strain and stress states of crenels perfectly corresponds to a uniaxial tensile state, this proves that the tension-compression hypothesis in the crenels is perfectly verified.

Nevertheless, for an angle of $2 \alpha=115^{\circ}$, the results are quite different. First, the loading state is not the same at the top and at the bottom of the crenel by comparing Fig. 9(b) and (d). Second, these two strain states do not correspond to uniaxial extension state but to a multiaxial state. The angles $2 \alpha=135^{\circ}$ and $2 \alpha=155^{\circ}$ (not presented here for a sake of clarity) present the same type of profile except that the crenels are loaded in compression instead of tension, which can lead to some buckling phenomenon. That means that other loadings than tension compression are present in the REV.

The presented results prove that the model hypothesis are totally valid for angles $2 \alpha=25^{\circ}, 2 \alpha=45^{\circ}$ and $2 \alpha=65^{\circ}$ whereas some errors appear for larger angles. These errors between the analytical model and the experimental results are explained by the multiaxial loading state of the crenels. That proves that for large angles the hypothesis to neglect coupling terms between crenels and the flat membranes become penalising for the modelling. Nevertheless, the performances of the model are quite encouraging for a formulation that do not add any material parameter that those of the bulk material.

\subsection{Abilities of the finite element model}

The estimated values of the components $\tilde{T}_{11}$ and $\tilde{T}_{22}$ are reported in Fig. 10 for all the investigated angles $2 \alpha$, versus the tensile elongation and are compared to the experimental values. This figure shows that whatever the considered mesostructure, the numerical simulations provide fairly good estimations of the experiments. Firstly, whatever the tested angle $2 \alpha$, the lateral computed tensions $\tilde{T}_{22}$ are close to 0 , i.e. very small compared to the macroscopic tensions $\tilde{T}_{11}$ computed along the tensile direction. It is verified that the experimental transformation gradients $\tilde{\mathbf{F}}$ applied to the simulations lead to tension states $\tilde{\mathbf{T}}$ corresponding to pure tensile mechanical loadings. Moreover, Fig. 10 also shows that the experimental and the simulated values of the tensions $\tilde{T}_{11}$ are close and satisfactory. Observed differences can be partially due to the rather simple model used to fit the silicone rubber behaviour. The largest error is observed for $2 \alpha=135^{\circ}$ and $2 \alpha=155^{\circ}$. A possible cause of such a discrepancy could be the poor scale separation observed in these two tests, leading to a poorer accuracy of the homogenised solution: indeed, for the technical reasons mentioned previously, only five and three REV could be conserved along the width of the tested specimen, respectively.

\section{Conclusion}

The paper presented a procedure to elaborate highly deformable architectured membranes made up of a hyperelastic silicone rubber and based on two different lattices of crenels positioned onto the upper and lower surfaces of the membranes. The orientation of the crenels was controlled during the injection moulding of uncured silicone rubber by using a special apparatus. Tensile tests were realised on the crenellated membranes allowing the analysis of the membranes mechanical behaviour. In particular, it was shown that by simply tuning the relative angle between the crenels, a wide range of mechanical behaviour could be obtained. In order to capture most of these mechanical features in a macroscopic model, the micromechanics of the membrane was upscaled by using a homogenisation method. Doing so, the macroscopic properties and momentum balance equations of the architectured membranes could be identified, together with proper localisation problems that were numerically solved in periodic REV in order to compute the effective properties of the architectured membranes. The comparison of the numerical predictions with the tensile experiments was satisfactory. In order to speed up macroscale simulations (e.g. for biomedical applications like artificial muscles) or to optimise the membrane architecture at the mesoscale (given a set of macroscale constraints), a simple analytical model based on both the behaviour of the tested silicone rubber and the architecture of the studied membranes was proposed to describe their macroscopic behaviour. Despite its simplicity, this model represents quite well the global behaviour of the structure and is as efficient as the upscaling method. Its main advantage is that it does not add any more parameter than the parameters of the constitutive equation of the silicone rubber and those describing the membrane mesoscale geometry. Moreover, it can easily be used in an optimisation process as it is not time consuming.

\section{Appendix A. 2D representation of the tensor}

For any deformation of the membrane, the deformation gradient can be written as:

$\mathbf{F}=\left[\begin{array}{ccc}F_{11} & F_{12} & 0 \\ F_{21} & F_{22} & 0 \\ 0 & 0 & F_{33}\end{array}\right]$

The restriction of the gradient to $2 \mathrm{D}$ formulation leads to:

$\tilde{\mathbf{F}}=\left[\begin{array}{ll}F_{11} & F_{12} \\ F_{21} & F_{22}\end{array}\right]$

The third invariant can be defined for the two tensors:

$\tilde{J}=\operatorname{det}(\tilde{\mathbf{F}}) J=\operatorname{det}(\mathbf{F})=\tilde{J} F_{33}$

The first invariant is also defined as:

$\widetilde{I_{1}}=\operatorname{tr}(\tilde{\mathbf{B}}) I_{1}=\operatorname{tr}(\mathbf{B})=\widetilde{I_{1}}+F_{33}^{2}=\widetilde{I}_{1}+\frac{1}{\tilde{J}^{2}}$

\section{Appendix B. Property of the first order displacement field $\mathbf{u}^{(0)}$}

By introducing the notation $\mathcal{F}^{(i)}=\varepsilon^{-1} \mathbf{F}^{(i)}$, or similarly $u^{(i)}=\varepsilon^{-1} \mathbf{u}^{(i)}$, the expansion (18) of $\mathbf{F}$ can be recasted as:

$\mathbf{F}=\mathcal{F}^{(-1)}+\varepsilon \mathcal{F}^{(0)}+\varepsilon^{2} \mathcal{F}^{(1)}+\ldots$

By using the Taylor expansion of $W$ around $\mathcal{F}^{(-1)}, W$ can be expressed as:

$W(\mathbf{F})=W^{(-1)}+\varepsilon W^{(0)}+\ldots$

with 


$$
\left\{\begin{array}{l}
W^{(-1)}=W\left(\mathcal{F}^{(-1)}\right) \\
W^{(0)}=\frac{\partial W}{\partial \mathcal{F}^{(-1)}}: \mathcal{F}^{(0)} \\
\cdots
\end{array}\right.
$$

Similarly, the Taylor expansion of $\pi$ around $\mathcal{F}^{(-1)}$ shows that $\pi$ can be written as:

$\pi(\mathbf{F})=\pi^{(-1)}+\varepsilon \pi^{(0)}+\ldots$

with

$$
\left\{\begin{array}{l}
\pi^{(-1)}=\pi\left(\mathcal{F}^{(-1)}\right)=\frac{\partial W}{\partial \mathcal{F}^{(-1)}} \\
\pi^{(0)}=\frac{\partial \pi}{\partial \mathcal{F}^{(-1)}}: \mathcal{F}^{(0)} \\
\ldots
\end{array} .\right.
$$

Therefrom, at the highest order, the boundary value problem to be solved within the REV therefore reads:

$$
\left\{\begin{array}{ll}
\operatorname{Div}_{Y} \pi^{(-1)}=0 \\
\pi^{(-1)}=\frac{\partial W}{\partial \mathcal{F}^{(-1)}}
\end{array}\right\} \text { in } \Omega_{R E V}
$$

where $\mathcal{U}^{(0)}=\varepsilon^{-1} \mathbf{u}^{(0)}$ is the unknown field. Accounting for the boundary condition (third equation of B.6) and the periodicity of $u^{(0)}$, the weak formulation of the above problem is

$$
\int_{\Omega_{R E V}} \frac{\partial W}{\left.\partial \mathcal{F}^{(-1}\right): \operatorname{Grad}_{Y} \mathbf{u}^{*} \mathrm{~d} V_{0}=0}
$$

where $\mathbf{u}^{*}$ is a virtual displacement field. By choosing $\mathbf{u}^{*}=\mathrm{d} u^{(0)}$, the last formulation becomes:

$$
\int_{\Omega_{R E V}} \mathrm{~d} W\left(\mathcal{F}^{(-1)}\right) \mathrm{d} V_{0}=0
$$

or

$$
\mathrm{d} \mathcal{W}\left(\mathcal{C}^{(-1)}\right)=0
$$

Such a relation holds whatever the considered deformed configuration $\mathcal{C}^{(-1)}$. From (13), it is concluded that $\mathcal{C}^{(-1)} \equiv \mathcal{C}_{0}$. As all material points of the REV are assumed to be undeformed and stress-free in the initial configuration, the last equivalence is possible only if:

$$
\mathcal{F}^{(-1)}=\mathbf{I},
$$

in absence of any rigid body motion, for all material points belonging to $\Omega_{R E V}$, so that

$$
\operatorname{Grad}_{Y} \mathbf{u}^{(0)}=\varepsilon \mathbf{I}
$$

As $\varepsilon \rightarrow 0$, the right hand side of the last expression vanishes, showing that the displacement field $\mathbf{u}^{(0)}$ does not depend on the space variable $\mathbf{Y}$ :

$\mathbf{u}^{(0)}=\mathbf{u}^{(0)}(\tilde{\mathbf{X}})$
This result allows us to recast the previous asymptotic expansions:

(i) Displacement field:

$\mathbf{u}=\mathbf{u}^{(0)}(\tilde{\mathbf{X}})+\varepsilon \mathbf{u}^{(1)}(\tilde{\mathbf{X}}, \mathbf{Y})+\ldots$

(ii) Gradient of the displacement

$\operatorname{Grad}_{X} \mathbf{u}=\operatorname{Grad}^{(0)} \mathbf{u}+\varepsilon_{\varepsilon} \operatorname{Grad}^{(1)} \mathbf{u}+\ldots$

by noting $\left\{\begin{array}{c}\operatorname{Grad}^{(0)} \mathbf{u}=\operatorname{Grad}_{Y} \mathbf{u}^{(1)}+\widetilde{\operatorname{Grad}_{\tilde{X}}} \mathbf{u}^{(0)} \\ \operatorname{Grad}^{(1)} \mathbf{u}=\operatorname{Grad}_{Y} \mathbf{u}^{(2)}+\widetilde{\operatorname{Grad}_{\tilde{X}}} \mathbf{u}^{(1)} \\ \ldots\end{array}\right.$

(iii) Gradient of the transformation

$\mathbf{F}=\mathbf{F}^{(0)}+\varepsilon \mathbf{F}^{(1)}+\ldots$

by noting $\left\{\begin{array}{l}\mathbf{F}^{(0)}=\mathbf{I}+\mathbf{G r a d}^{(0)} \mathbf{u} \\ \mathbf{F}^{(1)}=\mathbf{G r a d}^{(1)} \mathbf{u} \\ \ldots\end{array}\right.$

(iv) Strain energy function

$W(\mathbf{F})=W^{(0)}+\varepsilon W^{(1)}+\ldots$

with $\left\{\begin{array}{l}W^{(0)}=W\left(\mathbf{F}^{(0)}\right) \\ W^{(1)}=\frac{\partial W}{\partial \mathbf{F}^{(0)}}: \mathbf{F}^{(1)} . \\ \ldots\end{array}\right.$

(v) Stress tensor

$\pi(\mathbf{F})=\pi^{(0)}+\varepsilon \pi^{(1)}+\ldots$

with $\left\{\begin{array}{l}\pi^{(0)}=\pi\left(\mathbf{F}^{(0)}\right)=\frac{\partial W}{\partial \mathbf{F}^{(0)}} \\ \pi^{(1)}=\frac{\partial \pi}{\partial \mathbf{F}^{(0)}}: \mathbf{F}^{(1)} \\ \ldots\end{array}\right.$

Therefrom, such expansions are re-introduced in the problem and the identification process is re-iterated. Such a procedure provides the self equilibrium of the REV (Appendix C), which is required to estimate quantitatively the effective macroscopic mechanical properties of the membrane, the macroscopic momentum balance equations of the membrane (Appendix D) and the structure and the properties of its macroscopic mechanical behaviour (Appendix E).

\section{Appendix C. Self equilibrium of the REV}

At the zero order, the identification process yields to a boundary value problem, usually named as the self equilibrium of the REV: 


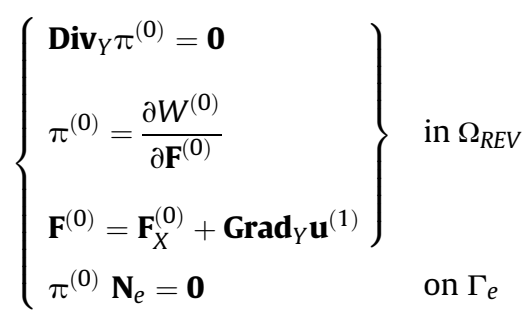

where $\mathbf{F}_{X}^{(0)}=\mathbf{I}+\widetilde{\operatorname{Grad}_{X} \mathbf{u}^{(0)}}$ is given and constant within the whole REV and where the unknown $\mathbf{u}^{(1)}$ is periodic. Its corresponding weak formulation is:

$$
\int_{\Omega_{R E V}} \pi^{(0)}: \operatorname{Grad}_{Y} \mathbf{u}^{*} \mathrm{~d} V_{0}=0
$$

where $\mathbf{u}^{*}$ is a kinematically admissible displacement field. Solving such a boundary value problem allows the calculation of the stress tensor $\pi^{(0)}$, and hence the effective properties of the equivalent continuum to be estimated.

\section{Appendix D. Macroscopic momentum balance equations}

The first momentum balance equation which is identified at the $\varepsilon$-order is expressed as:

$\widetilde{\operatorname{Div}}_{\tilde{X}} \pi^{(0)}+\operatorname{Div}_{Y} \pi^{(1)}=0$.

By integrating the last equation over $\Omega_{R E V}$, by applying the divergence theorem on the second term of the left hand-side, and by accounting for both the periodicity condition and the boundary condition:

$$
\pi^{(1)} \mathbf{N}_{e}=0 \text { on } \Gamma_{e},
$$

Equation (D.1) yields to the following macroscopic 2D balance equation:

$$
\widetilde{\operatorname{Div}}_{\tilde{X}} \tilde{\mathbf{T}}=\tilde{0}
$$

where the first order macroscopic in-plane tension $\tilde{\mathbf{T}}$ is defined as:

$\tilde{\mathbf{T}}=\frac{1}{S_{0}} \int_{\Omega_{R E V}} \tilde{\pi}^{(0)} \mathrm{d} V_{0}$

Furthermore, at the zero order, the second momentum balance equation becomes:

$\pi^{(0)} \mathbf{F}^{(0) t}=\mathbf{F}^{(0)} \pi^{(0) t}$

As previously, by integrating the last equation over $\Omega_{R E V}$, by applying the divergence theorem, and by accounting for both the periodicity condition and the boundary condition (C.1c), it is possible to obtain, after some calculations:

$\tilde{\mathbf{T}} \tilde{\mathbf{F}}^{t}=\tilde{\mathbf{F}} \tilde{\mathbf{T}}^{t}$

where

$$
\tilde{\mathbf{F}}=\tilde{\mathbf{I}}+\widetilde{\operatorname{Grad}_{X}} \tilde{\mathbf{u}}^{(0)}
$$

represents the macroscopic in-plane transformation gradient.

\section{Appendix E. Macroscopic constitutive equations}

At the first order, the constitutive Equation (C.1b) can be expressed in a differential form:

$\mathrm{d} W^{(0)}=\pi^{(0)}: \mathrm{d} \mathbf{F}^{(0)}$

or, by taking into account the definition (B.17a) of $\mathbf{F}^{(0)}$ :

$\mathrm{d} W^{(0)}=\tilde{\pi}^{(0)}: \widetilde{\mathrm{d} F}+\pi^{(0)}: \operatorname{Grad}_{Y} \mathrm{~d} \mathbf{u}^{(1)}$

The integration of the last equation over $\Omega_{R E V}$ yields to:

$\mathrm{d} \tilde{\mathcal{W}}=\tilde{\mathbf{T}}: \widetilde{\mathrm{d} F}+\frac{1}{S_{0}} \int_{\Omega_{R E V}} \pi^{(0)}: \operatorname{Grad}_{Y} \mathrm{~d} \mathbf{u}^{(1)} \mathrm{d} V_{0}$

where:

$\tilde{\mathcal{W}}=\frac{1}{S_{0}} \int_{\Omega_{R E V}} W^{(0)} \mathrm{d} V_{0}$

is the first order strain energy function of REV per unit of initial thickness. Choosing $\mathbf{u}^{*}=\mathbf{d u}^{(1)}$ in (C.2) shows that the last term of the right hand side of (E.3) vanishes so that $\tilde{\mathcal{W}}$ only depends on the in-plane macroscopic transformation gradient $\tilde{\mathbf{F}}$. Consequently, at the first order, the overall in-plane behaviour of the REV, i.e. its macroscopic in-plane behaviour, is hyperelastic. Its associated macroscopic in-plane strain energy function is $\tilde{\mathcal{W}}$, and its corresponding first order macroscopic in-plane tension obeys to the following macroscopic in-plane hyperelastic constitutive equation:

$\tilde{\mathbf{T}}=\frac{\partial \tilde{\mathcal{W}}}{\partial \tilde{\mathbf{F}}}$

\section{References}

Ashby, M., 2013. Designing architectured materials. Scr. Mater. 68, 4-7. Bensoussan, A., Lions, J.-L., Papanicolaou, G., 1978. Asymptotic Analysis for Periodic Structures. North Holland, Amsterdam.

Bouaziz, O., Brechet, Y., Embury, J.D., 2008. Heterogeneous and architectured materials: a possible strategy for design of structural materials. Adv. Eng. Mater. 10 24-36.

Bouaziz, O., 2013. Geometrically induced strain hardening. Scr. Mater. 68, 28-30.

Bréchet, Y., Embury, J.D., 2013. Architectured materials: expanding materials space. Scr. Mater. 68, 1-3.

Chen, Q.Z., Harding, S.E., Ali, N.N., Lyon, R.A., Boccaccini, A.R., 2008. Biomaterials in cardiac tissue engineering: ten years of research survey. Mater. Sci. Eng. 59, 1-37.

Dunlop, J.W.C., Brechet, Y.J.M., 2009. Architecture structural materials: a parallel between nature and engineering. Archit. Multifunct. Mater. 1188, 15-25.

Fleck, N.A., Deshpande, V.S., Ashby, M.F., 2010. Micro-architectured materials: past, present and future. Proc. R. Soc. Lond. A 466, 2495-2516.

Gibson, L.J., Ashby, M.F., 1982. The mechanics of three dimensional cellular materials. Proc. R. Soc. Lond. A 382, 43-59.

Gibson, L.J., Ashby, M.F., 1988. Cellular Solids: Structure and Properties. Pergamon Press.

Hard af Segerstad, P., Larsson, R., Toll, S., 2008. A constitutive equation for open-cel cellular solids, including viscoplasticity, damage and deformation induced anisotropy. Int. J. Plast. 24, 896-914.

Holzapfel, G.A., Gasser, T.C., Ogden, R.W., 2000. A new constitutive framework for arterial wall mechanics and a comparative study of material models. J. Elast. 61, $1-48$.

Jacobsen, A.J., Barvosa-Carter, W., Nutt, S., 2008. Shear behavior of polymer microscale truss structures formed from self-propagating polymer waveguides. Acta Mater. 56, 1209-1218.

Kidoaki, S., Kwon, I.K., Matsuda, T., 2008. Mesoscopic spatial designs of nano- and microfiber meshes for tissue-engineering matrix and scaffold based on newly devised multilayering and mixing electrospinning techniques. Biomaterials 26, $37-46$.

Laszczyk, L., Dendievel, R., Bouaziz, O., Brechet, Y., Parry, G., 2008. Design of architectured sandwich core materials using topological optimization methods. Archit. Multifunct. Mater. 1188, 131-136.

Ma, P.X., 2008. Biomimetic materials for tissue engineering. Adv. Drug Deliv. Rev. 60, 184-198. 
Machado, G., Chagnon, G., Favier, D., 2010. Analysis of the isotropic models of the Mullins effect based on filled silicone rubber experimental results. Mech. Mater. $42,841-851$.

Machado, G., Favier, D., Chagnon, G., 2012a. Determination of membrane stress-strain full fields of bulge tests from SDIC measurements. Theory, validation and experimental results on a silicone elastomer. Exp. Mech. 52, 865-880.

Machado, G., Chagnon, G., Favier, D., 2012b. Induced anisotropy by the Mullins effect in filled silicone rubber. Mech. Mater. 50, 70-80.

Melchels, F.P., Bertoldi, K., Bertoldi, R., Velders, A.H., Feijen, J., Grijpma, D.W., 2010. Mathematically defined tissue engineering scaffold architectures prepared by stereolithography. Biomaterials 31, 6909-6916.

Meunier, L., Chagnon, G., Favier, D., Orgéas, L., Vacher, P., 2008. Mechanical experimental characterisation and numerical modelling of an unfilled silicone rubber. Polym. Test. 27, 765-777.

Orteu, J.J., 2009. 3-D computer vision in experimental mechanics. Opt. Laser Eng. 47, $282-291$.

Patterson, J., Martino, M.M., Hubbell, J.A., 2010. Biomimetic materials in tissue engineering. Mater. Today 13, 14-22.

Pouget, E., Tonnar, J., Lucas, P., Lacroix-Desmazes, P., Ganachaud, F., Boutevin, B., 2010. Well-architectured poly(dimethylsiloxane)-containing copolymers obtained by radical chemistry. Chem. Rev. 110, 1233-1277.
Pruchnicki, E., 1998a. Hyperelastic homogenized law for reinforced elastomer at finite strain with edge effects. Acta Mech. 129, 139-162.

Pruchnicki, E., 1998b. Homogenized nonlinear constitutive law using Fourier series expansion. Int. J. Solids Struct. 135, 1895-1913.

Rey, T., Chagnon, G., Le Cam, J.-B., Favier, D., 2013. Influence of the temperature on the mechanical behaviour of filled and unfilled silicone rubbers. Polym. Test. 32 492-501.

Sanchez-Palencia, E., 1980. Non-homogeneous Media and Vibration Theory. Lecture Notes in Physics. Springer-Verlag, Berlin, Germany.

Spencer, A.J.M., 1971. Theory of Invariants, Continuum Physics, C. Eringen. Academic Press.

Talbi, N., Batti, A., Ayad, R., Guo, Y.Q., 2009. An analytical homogenization model for finite element modelling of corrugated cardboard. Compos. Struct. 88, 280-289.

Toll, S., 1998. Packing mechanics of fiber reinforcements. Polym. Eng. Sci. 38, $1337-1350$

Treloar, L.R.G., 1943. The elasticity of a network of long chain molecules (I and II). Trans. Faraday Soc. 39, 36-64; 241-246.

Vacher, P., Dumoulin, S., Morestin, F., Mguil-Touchal, S., 1999. Bidimensional strain measurement using digital images. Proc. Mech. Eng. C 213, 811-817.

Yeoh, O.H., 1990. Characterization of elastic properties of carbon black filled rubber vulcanizates. Rubber Chem. Technol. 63, 792-805. 